\title{
MINIMIZING OPPORTUNITY COST IN SELECTING THE BEST FEASIBLE DESIGN
}

\author{
Nugroho A. Pujowidianto \\ Hewlett-Packard Singapore \\ 138 Depot Road \\ Singapore 109683, SINGAPORE
}

\author{
Loo Hay Lee \\ Industrial \& Systems Engineering \\ National University of Singapore \\ 10 Kent Ridge Crescent \\ Singapore 119260, SINGAPORE
}

\author{
Chun-Hung Chen \\ Systems Engineering \& Operations Research \\ George Mason University \\ 4400 University Drive, MS 4A6 \\ Fairfax, VA 22030, USA
}

\begin{abstract}
Constrained ranking and selection $(\mathrm{R} \& S)$ refers to the problem of selecting the best feasible design where both main objective and constraint measures need to be estimated via stochastic simulation. Despite the growing interests in constrained $\mathrm{R} \& \mathrm{~S}$, none has considered other selection qualities than a statistical measure called the probability of correct selection (PCS). In contrast, several new developments in other $\mathrm{R} \& \mathrm{~S}$ literatures have considered financial significance as the selection quality. This paper aims to lay the foundation of using other selection qualities by attempting to minimize the opportunity cost in allocating the limited simulation budget. The opportunity cost is defined and two allocation rules which minimize its upper bound are presented together with a fully-sequential heuristic algorithm for implementation.
\end{abstract}

\section{INTRODUCTION}

We consider a constrained optimization where the number of alternatives is fixed. Due to uncertainties involved and the dynamic relationship between the parameters, both the main objective and constrained measures are estimated via stochastic simulation. As there is a finite number of alternatives, this problem falls under the Ranking and Selection $(R \& S)$ literatures. For introduction to R\&S, excellent reviews are provided by Swisher et al. (2003), Kim and Nelson (2006), and Hong and Nelson (2009) while the examples of the problems can be found at www.simopt.org (Pasupathy and Henderson 2006; 2011). The goal of the initial works is to guarantee the desired level of probability of correct selection (PCS). This is done by determining the number of allocation needed or developing stopping rules which can reach more than the user-specified level of PCS when the procedure stops.

There is also another stream of works which attempt to optimize the simulation budget. This is because the use of simulation requires considerable simulation efforts. Therefore instead of looking at the worst-case scenario, it develops a $R \& S$ procedure for maximizing the desired selection quality such as PCS using an average-case analysis. The resulting procedures are thus generally more efficient (Branke et al. 2007).

There have been many recent developments on R\&S with multiple performance measures to complement the R\&S literatures which initially focus on the unconstrained case (Rinott 1978, Kim and Nel- 
son 2001, Chen et al. 2000). The problems with multiple performance measures can be classified into two groups. If all multiple performance measures are equally important, the problem can be modeled as multiobjective optimization. Examples of simulation budget allocation procedures for multi-objective R\&S can be found in Butler et al. (2001) which guarantee PCS or in Lee et al. (2010) and Teng et al. (2010) which maximize $P C S$. In some cases, it is more appropriate to use the constrained R\&S model where there is one primary performance measure as the main objective while the rest of the secondary performance measures can be treated as constrained measures.

Despite the recent interests, all of the constrained R\&S procedures focus only on PCS. Andradóttir et al. (2005) and Andradóttir and Kim (2010) laid the foundation of constrained R\&S literatures by developing several procedures for guaranteeing PCS when there is a single constraint. The procedures consist of the feasibility determination phase and the selection phase which can be implemented either sequentially or simultaneously. For the case with multiple performance measures, the feasibility determination can be made more efficient as shown in Batur and Kim (2010). Integrating the two works yield in a valid procedure for selecting the best feasible design as proposed by Healey et al. (2013a). Healey et al. (2013b) and Healey et al. (2013c) provided some extensions by letting some designs to be dormant from the sampling process temporarily and by handling the issues in switching between alternatives. Another alternative is to convert the constrained R\&S into its unconstrained version using multi-attribute utility theory as proposed by Morrice and Butler (2006). As all these procedures focus on guaranteeing PCS, there have also been some efforts for maximizing PCS in constrained R\&S. Kabirian and Olafsson (2009) developed a heuristic algorithm based on the feasibility and optimality indicators without studying PCS analytically. Pujowidianto et al. (2009) and Lee et al. (2012) provided a closed-form allocation rule which asymptotically maximizes the lower bound of PCS that is developed using Bonferroni inequalities. The Bonferroni approximation is applicable for both the case of independent performance measures and the case where the performance measures are correlated. Hunter and Pasupathy $(2010,2012)$ attempted to maximize the PCS using large-deviations perspective by minimizing the rate of the probability of false selection $(P F S, P F S=1-P C S)$ for the case with independent performance measures. None of the previously mentioned works explicitly account for the correlation between the main objective and the constrained measures. This is handled by Hunter et al. (2011) and Pujowidianto et al. (2012).

In some cases, the PCS is unable to represent the decision maker's interest. This is because the decision maker may be more concerned of economic criteria than statistical significance. For example, 10\% PCS with $\$ 1$ loss for every false selection may be preferred to $90 \%$ PCS with $\$ 1$ million loss in the event that false selection occurs. This concept was first addressed by Chick and Inoue (2001) by minimizing the expected opportunity cost $(E O C)$ in the context of selecting the best in an unconstrained optimization. Lee et al. (2007) provided a procedure for minimizing the opportunity cost of the observed Pareto and nonPareto set for a multi-objective R\&S. However, none has used the opportunity cost $(O C)$ concept in a constrained R\&S. Table 1 summarizes the development of R\&S and the gap in the context of constrained R\&S.

This paper aims to lay the foundation of constrained R\&S based on economic criteria by minimizing the upper bound of $O C$. The insights on the difference between the allocation rule when the criteria is $O C$ and when PCS is the concern are discussed. This is a useful contribution which will lead the development of other simulation budget allocation procedures which focus on the financial significance. For example, in the context of unconstrained optimization, Chick et al. (2010) attempted to maximize the expected value of information of individual alternative if there can only be one sample to be allocated in the next stage of sampling process. Chick and Gans (2009) and Chick and Frazier (2012) pioneered the modeling of the simulation budget allocation procedure which looks at the stream of expected rewards. Therefore, instead of myopically allocate the budget via one-step lookahead, they consider the overall rewards using dynamic programming. 
Table 1: Overview of R\&S and the Research Gap in Constrained R\&S

\begin{tabular}{|c|c|c|c|}
\hline & \multirow{2}{*}{$\begin{array}{c}\text { Guaranteeing } \\
\text { Desired } P C S\end{array}$} & \multicolumn{2}{|c|}{ Maximizing Desired Selection Quality } \\
\hline & & $P C S$ & $O C$ \\
\hline Unconstrained optimization & $\begin{array}{l}\text { Rinott (1978), Kim } \\
\text { and Nelson (2001), } \\
\text { Nelson et al. (2001) }\end{array}$ & $\begin{array}{c}\text { Chen et al. (2000, } \\
\text { 2010) }\end{array}$ & $\begin{array}{c}\text { Chick and Inoue } \\
\text { (2001a, 2001b), } \\
\text { Chick and Wu } \\
(2005), \text { He et al. } \\
(2007)\end{array}$ \\
\hline Constrained optimization & $\begin{array}{l}\text { Andradóttir et al. } \\
\text { (2005), Andradóttir } \\
\text { and Kim (2010), } \\
\text { Morrice and Butler } \\
\text { (2006) }\end{array}$ & $\begin{array}{c}\text { Pujowidianto et } \\
\text { al. (2009, 2012), } \\
\text { Hunter and Pasu- } \\
\text { pathy (2010, } \\
\text { 2012), Lee et al. } \\
\text { (2012) }\end{array}$ & $?$ \\
\hline Multi-objective optimization & Butler et al. (2001) & $\begin{array}{l}\text { Lee et al. (2010), } \\
\text { Teng et al. (2010) }\end{array}$ & Lee et al. (2007) \\
\hline
\end{tabular}

The organization of this paper is as follows. Section 2 formulates the simulation budget allocation problem and defines the opportunity cost in the context of constrained optimization. Section 3 presents the allocation rules which minimize the upper bound of the total opportunity cost (TOC). The sequential heuristic algorithm for implementation is provided in Section 4. Section 5 concludes this paper.

\section{PROBLEM FORMULATION}

\subsection{Selection Problem}

We consider the problem of selecting the best feasible design from $k$ alternatives. Let $X_{i}$ be the random variable of the output of the main objective of design $i$ while $Y_{i l}$ is the random variable of the output of the $l$ th constraint measure, $, i=1,2, \ldots, k, l=1,2, \ldots, s . X_{i j}$ and $Y_{i l j}$ are the $j$ th replications of $X_{i}$ and $Y_{i l}$ respectively. The main objective value is $h_{i}=E\left[X_{i j}\right]$ and the $l$ th constraint measure value is $g_{i l}=$ $E\left[Y_{i l j}\right]$. The variances are $\sigma_{h_{i}}^{2}=\operatorname{Var}\left[X_{i j}\right]$ and are $\sigma_{g_{i l}}^{2}=\operatorname{Var}\left[Y_{i l j}\right]$. We assume that the performance measures are normally distributed. The output is independent from design to design as well as from replication to replication. In addition, the performance measures are independent from one to another.

Without loss of generality, the goal of the selection problem is to select the design with the smallest main objective value where all of the constraint measures should be below the respective constraint limits $\gamma_{l}$. The constrained R\&S problem is therefore to select the best feasible design $b$ as follows

$$
b=\arg \min _{i=1,2, \ldots, k} h_{i} \text { subject to } g_{i l} \leq \gamma_{l}, l=1,2, \ldots, s .
$$

In practice, the performance measures need to be estimated via simulation. Let $n_{i}$ be the number of replications for design $i$. Thus, the performance measures can be estimated by the sample means, $\widehat{H}_{i}=\bar{x}_{i}=$ $\sum_{j=1}^{n_{i}} X_{i j} / n_{i}$ and $\hat{G}_{i l}=\bar{y}_{i l}=\sum_{j=1}^{n_{i}} Y_{i l j} / n_{i}$. The selection is based on the sample mean and so the selected alternative $\mathfrak{D}$ is

$$
\mathfrak{D}=\arg \min _{i=1,2, \ldots, k} \widehat{H}_{i} \text { subject to } \widehat{G}_{i l} \leq \gamma_{l}, l=1,2, \ldots, s .
$$




\subsection{Simulation Budget Allocation Problem}

Let $\beta$ be the simulation budget. The objective is to minimize the total opportunity cost (TOC) and so the simulation budget allocation problem is

$$
\min _{n_{1}, n_{2}, \ldots, n_{k}} \text { TOC subject to } \sum_{i=1}^{k} n_{i}=\beta, n_{i} \geq 0 .
$$

\subsection{Definition of Opportunity Cost $(O C)$}

As there are multiple performance measures, there are several opportunity costs. First, there is an opportunity cost in terms of the main objective if the selected alternative is inferior to the best feasible design. Secondly, there is an opportunity cost for accounting the loss by selecting an infeasible design as the best.

Let $O C_{h}$ be the opportunity cost in terms of main objective. The opportunity cost is the difference in means of the main objective values between the selected alternative and the best as follows

$$
O C_{h_{\mathfrak{D}}}=\max \left(h_{\mathfrak{D}}-h_{b}, 0\right)
$$

In addition, let $O C_{g_{l}}$ be the opportunity cost in terms of the $l$ th constraint measure. The opportunity cost is the difference between the mean of the constraint measure with the respective constraint limit multiplied by weight factors $\omega_{l}, l=1,2, \ldots, s$. The weight factor is used to monetize the penalty of violating the constraint measures. For example, if the goal is to minimize the total operations cost while ensuring the average waiting time is less than certain limit, the weight factor would be the cost of the excess in waiting time.

$$
O C_{g_{\mathfrak{D} l}}=\omega_{l} \max \left(g_{\mathfrak{D} l}-\gamma_{l}, 0\right)
$$

If appropriate, $O C_{g_{\mathfrak{D} l}}$ can be modified into a step function. This is useful when there is different penalties of violating the constraint limit for each specific ranges.

The total opportunity cost (TOC) is therefore

$$
T O C=\sum_{i \neq b}\left\{\left[\left(O C_{h_{i}}+\sum_{l=1}^{s} O C_{g_{i l}}\right) \mid \mathfrak{D}=i\right] P\{\mathfrak{D}=i\}\right\},
$$

where

$$
P\{\mathfrak{D}=i\}=P\left\{\bigcap_{l=1}^{s}\left(\widehat{G}_{i l} \leq \gamma_{l}\right) \cap\left[\bigcap_{j \neq i}\left[\bigcup_{l=1}^{s}\left(\widehat{G}_{j l}>\gamma_{l}\right) \cup\left(\widehat{H}_{i}<\widehat{H}_{j}\right)\right]\right]\right\}
$$

Note that given a design is selected as the best, the total opportunity cost in (6) is the same as the case where the constraint measures are incorporated into the main objective to have an unconstrained optimization. However, the probability of design $i$ being the best is different. In this paper, a design can only be selected as the best if it is feasible with respect to all constraint limits. In the case where the decision maker converts the constraints into the main objective, there is a possibility that an infeasible design is the best design if it is close to the constraint limits and has a significantly better main objective than all feasible designs.

\section{ALLOCATION RULE}

This section provides some insights on the allocation rule that aims to minimize the estimated total opportunity cost as discussed in Section 3.1. For ease of illustration, Section 3.2 considers a special case with three designs when there is a single constraint. Two allocation rules are derived. Section 3.3 uses the asymptotic framework as the simulation budget goes to infinity, $\beta \rightarrow \infty$. This is because there are usually 
certain good properties when the problem is analyzed in the asymptotic context. Section 3.4 proposes an alternative allocation rule which considers the total opportunity cost in the next stage of sampling when all additional samples are allocated to only one design. Section 3.5 provides insights to generalize the results into the common scenarios with more than three designs in the presence of multiple constraints.

\subsection{Estimated Total Opportunity Cost (ETOC)}

There is no closed-form expressions for TOC in (6). It can be evaluated using Monte Carlo Simulation. However, we do not want to add the computational burden as the computational budget is limited. This is addressed by minimizing an upper bound to TOC called the estimated total opportunity cost (ETOC). The probability of design $i$ selected as the best can be bounded from above as follows

$$
P\{\mathfrak{D}=i\} \leq \min \left\{\min _{l} P\left(\widehat{G}_{i l} \leq \gamma_{l}\right), \min _{j \neq i} P\left[\bigcup_{l=1}^{S}\left(\widehat{G}_{j l}>\gamma_{l}\right) \cup\left(\widehat{H}_{i}<\widehat{H}_{j}\right)\right]\right\} .
$$

Assuming that the main objective and the constraint measures are independent,

$$
P\{\mathfrak{D}=i\} \leq \min \left\{\min _{l} P\left(\widehat{G}_{i l} \leq \gamma_{l}\right), \min _{j \neq i}\left[\sum_{l=1}^{S} P\left(\widehat{G}_{j l}>\gamma_{l}\right)+P\left(\widehat{H}_{i}<\widehat{H}_{j}\right)\right]\right\}
$$

Therefore, the estimated total opportunity cost ETOC is given by

$$
\begin{gathered}
\text { TOC } \leq \text { ETOC }=\sum_{i \neq b}\left\{\left(O C_{h_{i}}+\sum_{l=1}^{s} O C_{g_{l}}\right) \times \min \left\{\min _{l} P\left(\widehat{G}_{i l} \leq \gamma_{l}\right), \min _{j \neq i}\left[\sum_{l=1}^{S} P\left(\widehat{G}_{j l}>\gamma_{l}\right)+\right.\right.\right. \\
\left.\left.\left.P\left(\widehat{H}_{i}<\widehat{H}_{j}\right)\right]\right\}\right\} .
\end{gathered}
$$

The approximate simulation budget allocation rule becomes

$$
\min _{n_{1}, n_{2}, \ldots, n_{k}} \text { ETOC subject to } \sum_{i=1}^{k} n_{i}=\beta, n_{i} \geq 0
$$

\subsection{The Case with Three Designs}

Let design 1 be the best feasible design, i.e. $b=1$ where $h_{1}<\min _{i \neq 1} h_{i}$. As there is only one constraint, $g_{1 l}=g_{1} \leq \gamma$. Design 2 represents a design that is feasible but worse than the best design in terms of the main objective, i.e. $h_{1}<h_{2}, g_{2} \leq \gamma$. Design 3 has a better main objective value than the best design but it is infeasible, i.e. $h_{1}>h_{3}, g_{3}>\gamma$. The total opportunity cost (TOC) in this case becomes

$$
T O C=\left[\left(h_{2}-h_{1}\right) \mid \mathfrak{D}=2\right] P\{\mathfrak{D}=2\}+\left[\omega\left(g_{3}-\gamma\right) \mid \mathfrak{D}=3\right] P\{\mathfrak{D}=3\} .
$$

The idea of the upper bound of $P\{\mathfrak{D}=i\}$ is to look at the individual probability that is critical in determining its probability of being selected as the best. For example, design 2 is feasible while design 3 is infeasible. Therefore, there is a higher chance for design 2 to appear as feasible and to beat design 3 as design 3 is likely to be infeasible. In this case, the probability to be focused is the probability of design 2 beating the best design 1 , that is either when design 2 appears to be better than design 1 in terms of the main objective or design 1 appears to be infeasible. Similarly, design 3 has a high chance to appear to be better than designs 1 and 2 in terms of the main objective. Therefore, its probability of being the best is mostly influenced by its probability of being feasible. The estimated TOC (ETOC) can then be given by

$$
\text { ETOC }=\left[\left(h_{2}-h_{1}\right) \mid \mathfrak{D}=2\right]\left[P\left(\widehat{G}_{1}>\gamma\right)+P\left(\widehat{H}_{2}<\widehat{H}_{1}\right)\right]+\left[\omega\left(g_{3}-\gamma\right) \mid \mathfrak{D}=3\right] P\left(\widehat{G}_{3} \leq \gamma\right) .
$$


Let $\phi$ and $\Phi$ be the probability density function (pdf) and the cumulative distribution function (cdf) for the standard normal distribution, $\phi(t)=\frac{1}{\sqrt{2 \pi}} e^{-t^{2} / 2}, \Phi(t)=\int_{-\infty}^{t} \frac{1}{\sqrt{2 \pi}} e^{-u^{2} / 2} d u$. Given that $N\left(\mu, \sigma^{2}\right)$ denotes a normal distribution with mean $\mu$ and variance $\sigma^{2}$, let $f\left(x_{1,2}\right)$ be the pdf of $N\left(h_{1}-h_{2}, \hat{\sigma}_{h_{1,2}}^{2}\right)$, $\hat{\sigma}_{h_{1,2}}^{2}=\left(\frac{\sigma_{h_{1}}^{2}}{n_{1}}+\frac{\sigma_{h_{2}}^{2}}{n_{2}}\right), f\left(y_{1, \gamma}\right)$ be the pdf of $N\left(g_{1}-\gamma, \hat{\sigma}_{g_{1}}^{2}\right), \hat{\sigma}_{g_{1}}^{2}=\frac{\sigma_{g_{1}}^{2}}{n_{1}}, f\left(y_{\gamma, 3}\right)$ be the pdf of $N(\gamma-$ $\left.g_{3}, \hat{\sigma}_{g_{3}}^{2}\right)$, and $\hat{\sigma}_{g_{3}}^{2}=\frac{\sigma_{g_{3}}^{2}}{n_{3}}$. Therefore,

$$
\begin{aligned}
& {\left[\left(h_{2}-h_{1}\right) \mid \mathfrak{D}=2\right] P\left(\widehat{G}_{1}>\gamma\right)=\left(h_{2}-h_{1}\right) \int_{0}^{+\infty} f\left(y_{1, \gamma}\right) d y_{1, \gamma}=\left(h_{2}-h_{1}\right) \Phi\left(\frac{g_{1}-\gamma}{\widehat{\sigma}_{g_{1}}}\right),} \\
& {\left[\left(h_{2}-h_{1}\right) \mid \mathfrak{D}=2\right] P\left(\widehat{H}_{2}<\widehat{H}_{1}\right)=\left(h_{2}-h_{1}\right) \int_{0}^{+\infty} f\left(x_{1,2}\right) d x_{1,2}=\left(h_{2}-h_{1}\right) \Phi\left(\frac{h_{1}-h_{2}}{\widehat{\sigma}_{h_{1,2}}}\right),} \\
& {\left[\omega\left(g_{3}-\gamma\right) \mid \mathfrak{D}=3\right] P\left(\widehat{G}_{3} \leq \gamma\right)=\omega\left(g_{3}-\gamma\right) \int_{0}^{+\infty} f\left(y_{\gamma, 3}\right) d y_{\gamma, 3}=\omega\left(g_{3}-\gamma\right) \Phi\left(\frac{\gamma-g_{3}}{\widehat{\sigma}_{g_{3}}}\right),}
\end{aligned}
$$

and

$$
\text { ETOC }=\left(h_{2}-h_{1}\right)\left[\Phi\left(\frac{g_{1}-\gamma}{\widehat{\sigma}_{g_{1}}}\right)+\Phi\left(\frac{h_{1}-h_{2}}{\widehat{\sigma}_{h_{1,2}}}\right)\right]+\omega\left(g_{3}-\gamma\right) \Phi\left(\frac{\gamma-g_{3}}{\widehat{\sigma}_{g_{3}}}\right)
$$

Two of the possible ways to minimize ETOC are described in Sections 3.3 and 3.4. Section 3.3 shows how $E T O C$ can be asymptotically minimized while Section 3.4 aims to maximize the difference between ETOC before and after allocating some additional simulation budget to a design.

\subsection{Asymptotic Allocation}

The approximate simulation budget allocation problem can be solved by deriving the Karush-KuhnTucker (KKT) conditions to the problem in (11). By taking the natural log in solving the KKT conditions and letting the simulation budget tend to infinity, i.e. $\beta \rightarrow \infty$, only the terms inside $\Phi(\cdot)$ remain. In other words, the effect of the individual loss is dissolved. As a result, the allocation rule will be the same as that for maximizing the probability of correct selection PCS in Lee et al. (2012). The next section attempts to quantify the effect of the opportunity cost explicitly by looking at one step ahead.

\subsection{One-step Lookahead Allocation}

In this approach, we are interested in how to allocate additional samples $\tau_{i}$ to a single design $i$ given that $n_{i}$ samples have been observed for each design $i=1,2, \ldots, k$. This is similar to the approach by He et al. (2007) for unconstrained optimization. Given the additional sample to design $i, E T O C_{i}$ for the case with three designs can be updated as follows,

$$
\operatorname{ETOC}_{i}=\left(h_{2}-h_{1}\right)\left[\int_{0}^{+\infty} f^{*}\left(y_{1, \gamma}\right) d y_{1, \gamma}+f^{*}\left(x_{1,2}\right) d x_{1,2}\right]+\omega\left(g_{3}-\gamma\right) \int_{0}^{+\infty} f^{*}\left(y_{\gamma, 3}\right) d y_{\gamma, 3} .
$$


The idea is to update the variance of the pdf to consider the additional sample $\tau_{i}$. For example if $i=1, f^{*}\left(x_{1,2}\right)$ is the pdf of $N\left(h_{1}-h_{2}, \hat{\sigma}_{h_{1,2}}^{2}=\frac{\sigma_{h_{1}}^{2}}{n_{1}+\tau_{1}}+\frac{\sigma_{h_{2}}^{2}}{n_{2}}\right), f^{*}\left(y_{1, \gamma}\right)$ be the pdf of $N\left(g_{1}-\gamma, \hat{\sigma}_{g_{1}}^{2}=\right.$ $\left.\frac{\sigma_{g_{1}}^{2}}{n_{1}+\tau_{1}}\right)$ while there is no changes to $f\left(y_{\gamma, 3}\right)$ as $f^{*}\left(y_{\gamma, 3}\right)$ remains to be the pdf of $N\left(\gamma-g_{3}, \hat{\sigma}_{g_{3}}^{2}=\frac{\sigma_{g_{3}}^{2}}{n_{3}}\right)$. If $i=2$, only $f^{*}\left(x_{1,2}\right)$ is updated where $\hat{\sigma}_{h_{1,2}}^{2}=\frac{\sigma_{h_{1}}^{2}}{n_{1}}+\frac{\sigma_{h_{2}}^{2}}{n_{2}+\tau_{2}}$ while only $f^{*}\left(y_{\gamma, 3}\right)$ is updated if $i=3$ by changing the estimated variance $\hat{\sigma}_{g_{3}}^{2}=\frac{\sigma_{g_{3}}^{2}}{n_{3}+\tau_{3}}$.

The allocation rule is then to allocate the additional sample only to a design of which the difference between $E T O C_{i}$ and the current $E T O C$ is the highest.

\subsection{Generalization}

Sections 3.2 to 3.4 focus the discussion on the case with three designs when there is a single constraint. In general, there are often more than three designs and multiple constraints are present. The one-step lookahead allocation rule is expected to be similar. The presence of many constraints and designs will require one to compare the probabilities to derive the upper bound of the probability of being the best. This can be addressed by computing the respective opportunity costs. The probability to be focused is then the one that has the least opportunity cost so as to ensure that the upper bound of the TOC is tight.

\section{SEQUENTIAL ALGORITHM}

This section presents a fully-sequential heuristic algorithm for implementing the allocation rule. In practice, the means and variances can be estimated using sample mean and sample variance based on $n_{0}$ initial replications for all of the designs. Afterwards, the sampling budget is increased by $\tau=\sum_{i=1}^{k} \tau_{i}=1$. The algorithm is described as the following

INPUT number of designs $k$, the total simulation budget $\beta$, the initial number of replications $n_{0}$, the additional budget in each stage $\tau$, and;

INITIALIZE $\quad r \leftarrow 0$;

Perform $n_{0}$ samples for all designs; $n_{1, r}=n_{2, r}=\cdots=n_{k, r}=n_{0}$.

LOOP WHILE $\sum_{i=1}^{k} n_{i, r}<\beta$ DO

UPDATE Calculate sample means and sample standard deviation using the new simulation output; select the sample best feasible design $\mathfrak{D}$

ALLOCATE Increase the sampling budget by $\tau=1$ and select the design $i^{*}$ to receive the additional one sample

SIMULATE Perform additional one sample for design $i^{*}$; update $n_{i^{*}, r+1}=n_{i^{*}, r}+1 ; r \leftarrow r+1$. END OF LOOP

For the asymptotic allocation, the allocation rule is the same as that in Lee et al. (2012). In this case, $i^{*}=\arg \max _{i}\left(n_{i, r+1}-n_{i, r}\right)$ while $i^{*}=\arg \max _{i}\left|E T O C_{i}-E T O C\right|$ for the one-step lookahead allocation rule.

\section{CONCLUSION}

This paper revisits the problem of constrained ranking and selection where the performance metric of interest is the total opportunity cost instead of the probability of correct selection. This is to cater to the decision makers who are more interested in the economic value of the decision instead of the statistical significance. The problems are formulated and two possible allocation rules are presented. The asymptotic 
allocation turns out to be equivalent to that for probability of correct selection. Similar to the case of unconstrained optimization, the one-step lookahead allocation is able to consider the total opportunity cost by looking at each hypothetical value for allocating samples for a single design before the actual sampling. This research can be extended by considering the framework by Chick and Frazier (2012) which looks at the entire future stream of rewards instead of only one-step ahead. In addition, the empirical value of new allocation rules considering the total opportunity cost needs to be investigated.

\section{ACKNOWLEDGMENTS}

This work has been supported in part by National Science Foundation under Award CMMI-1233376, Department of Energy under Award DE-SC0002223, NIH under Grant 1R21DK088368-01, and National Science Council of Taiwan under Award NSC-100-2218-E-002-027-MY3. The authors would also like to thank the Proceedings Editors and two anonymous reviewers for their constructive feedbacks.

\section{REFERENCES}

Andradóttir, S., D. Goldsman, and S.-H. Kim. 2005. "Finding the best in the presence of a stochastic constraint." In Proceedings of the 2005 Winter Simulation Conference, ed. M. E. Kuhl, N. M. Steiger, F. B. Armstrong, and J. A. Joines, 732-738. Piscataway, New Jersey: Institute of Electrical and Electronics Engineers, Inc.

Andradóttir, S., and S.-H. Kim. 2010. "Fully Sequential Procedures for Comparing Constrained Systems via Simulation". Naval Research Logistics 57: 403-421.

Batur, D., and S.-H. Kim. 2010. "Finding Feasible Systems in the Presence of Constraints on Multiple Performance Measures". ACM Transactions on Modeling and Computer Simulation 20: 13:1-26.

Branke, J., S. E. Chick, and C. Schmidt. 2007. "Selecting a selection procedure." Management Science 53:1916-1932.

Butler, J., D. J. Morrice, and P. W. Mullarkey. 2001. "A multiple attribute utility theory approach to ranking and selection." Management Science 47:800-816.

Chen, C. H., J. Lin, E. Yücesan, and S. E. Chick. 2000. "Simulation budget allocation for further enhancing the efficiency of ordinal optimization." Discrete Event Dynamic Systems: Theory and Applications 10:251-270.

Chick, S., and P. Frazier. 2012. "Sequential Sampling with Economics of Selection Procedures." Management Science 58: 550 - 569.

Chick, S., and N. Gans. 2009. "Economic Analysis of Simulation Selection Problems." Management Science 55: $421-437$.

Chick, S. E., and K. Inoue. 2001. "New two-stage and sequential procedures for selecting the best simulated system." Operations Research 49: 732-743.

Chick SE, Y., and Z. Wu. 2005. "Selection Procedures with Frequentist Expected Opportunity Cost Bounds." Operations Research 53: 867-878.

Chick, S. E., J. Branke, and C. Schmidt. 2010. "Sequential sampling to myopically maximize the expected value of information." INFORMS Journal on Computing 22, 71-80.

He, D., S. E. Chick, and C. H. Chen. 2007. "The Opportunity Cost and OCBA Selection Procedures in Ordinal Optimization." IEEE Transactions on Systems, Man, and Cybernetics-Part C (Applications and Reviews) 37: 951-961.

Healey, C., S. Andradóttir, and S.-H. Kim. 2013a. "Selection Procedures for Simulations with Multiple Constraints under Independent and Correlated Sampling." Submitted.

Healey, C., S. Andradóttir, and S.-H. Kim. 2013b. "Efficient Comparison of Constrained Systems using Dormancy." European Journal of Operations Research 224:340-352

Healey, C., S. Andradóttir, and S.-H. Kim. 2013c. "Minimal Switching Procedures for Constrained Ranking and Selection under Independent and Common Random Numbers." Under Review 
Hong, L. J., and B. L. Nelson. 2009. "A Brief Introduction to Optimization via Simulation." In Proceedings of the 2009 Winter Simulation Conference, Edited by M. D. Rosetti, R. R. Hill, B. Johansson, A. Dunkin, and R. Ingalls, 75-85. Piscataway, New Jersey: Institute of Electrical and Electronics Engineers, Inc.

Hunter, S. R. and R. Pasupathy. 2010. "Large-deviation sampling laws for constrained simulation optimization on finite sets." In Proceedings of the 2010 Winter Simulation Conference, Edited by B. Johansson, S. Jain, J. Montoya-Torres, J. Hugan, and E. Yücesan, 995-1002. Piscataway, New Jersey: Institute of Electrical and Electronics Engineers, Inc.

Hunter, S. R. and R. Pasupathy. 2012. "Optimal sampling laws for stochastically constrained simulation optimization on finite sets." INFORMS Journal on Computing Articles in Advance, 1-16.

Hunter, S. R., N. A. Pujowidianto, C. H. Chen, L. H. Lee, R. Pasupathy, and C. M. Yap. 2011. "Optimal sampling laws for constrained simulation optimization on finite sets: the bivariate normal case." In Proceedings of the 2011 Winter Simulation Conference, edited by S. Jain, R. R. Creasey, J. Himmelspach, K. P. White, and M. Fu, 4294-4302. Piscataway, New Jersey: Institute of Electrical and Electronics Engineers, Inc.

Kabirian, A., and S. Ólafsson. 2009. "Selection of the best with stochastic constraints." In Proceedings of the 2009 Winter Simulation Conference, Edited by M. D. Rosetti, R. R. Hill, B. Johansson, A. Dunkin, and R. Ingalls, 574-583. Piscataway, New Jersey: Institute of Electrical and Electronics Engineers, Inc.

Kim, S. H., and B. L. Nelson. 2001. "A fully sequential procedure for indifference-zone selection in simulation." ACM Transactions on Modeling and Computer Simulation 11:251-273.

Kim, S. H., and B. L Nelson. 2006. "Selecting the Best System." In Handbook in Operations Research and Management Science: Simulation, Edited by S. Henderson and B. L. Nelson, Chapter 17, 501534. Elsevier, Amsterdam.

Lee, L. H., E. P. Chew, and S. Teng. 2007. "Finding the Pareto Set for Multi-objective Simulation Models by Minimization of Expected Opportunity Cost." In Proceedings of the 2007 Winter Simulation Conference, ed. S. G. Henderson, B. Biller, M.-H. Hsieh, J. Shortle, J. D. Tew, and R. R. Barton, 513521. Piscataway, New Jersey: Institute of Electrical and Electronics Engineers, Inc.

Lee, L. H., E. P. Chew, S. Teng, and D. Goldsman. 2010. "Finding the non-dominated Pareto set for multi-objective simulation models." IIE Transactions 42:656-674.

Lee, L. H., N. A. Pujowidianto, L.-W. Li, C. H. Chen, and C. M. Yap. 2012. "Approximate simulation budget allocation for selecting the best system in the presence of stochastic constraints." IEEE Transactions on Automatic Control 57: 2940-2945.

Morrice, D. J. and J. C. Butler. 2006. "Ranking and selection with multiple "targets". In Proceedings of the 2006 Winter Simulation Conference, ed. L. F. Perrone, F. P. Wieland, J. Liu, B. G. Lawson, D. M. Nicol, and R. M. Fujimoto, 222-230. Piscataway, New Jersey: Institute of Electrical and Electronics Engineers, Inc.

Pasupathy, R., and S. G. Henderson. 2006, December. "A Testbed of Simulation-Optimization Problems". In Proceedings of the 2006 Winter Simulation Conference, edited by L. F. Perrone, F. P. Wieland, J. Liu, B. G. Lawson, D. M. Nicol, and R. M. Fujimoto, 255-263. Piscataway, New Jersey: Institute of Electrical and Electronics Engineers, Inc.

Pasupathy, R., and S. G. Henderson. 2011, December. "SimOpt: A Library of Simulation Optimization Problems". In Proceedings of the 2011 Winter Simulation Conference, edited by S. Jain, R. R. Creasey, J. Himmelspach, K. P. White, and M. Fu, 4080-4090. Piscataway, New Jersey: Institute of Electrical and Electronics Engineers, Inc.

Pujowidianto, N. A., L. H. Lee, C. H. Chen, and C. M. Yap. 2009. "Optimal computing budget for constrained optimization." In Proceedings of the 2009 Winter Simulation Conference, Edited by M. D. Rosetti, R. R. Hill, B. Johansson, A. Dunkin, and R. Ingalls, 584-589. Piscataway, New Jersey: Institute of Electrical and Electronics Engineers, Inc. 
Pujowidianto, N. A., S. R. Hunter, R. Pasupathy, L. H. Lee, and C. H. Chen. 2012. "Closed-form sampling laws for stochastically constrained simulation optimization on large finite sets." In Proceedings of the 2012 Winter Simulation Conference, Edited by C. Laroque, J. Himmelspach, R. Pasupathy, O. Rose, and A. M. Uhrmacher, Article No. 14. Piscataway, New Jersey: Institute of Electrical and Electronics Engineers, Inc.

Rinott, Y. 1978. "On two-stage selection procedures and related probability inequalities." Communications in Statistics A7:799-811.

Swisher, J. R., S. H. Jacobson, and E. Yücesan. 2003. "Discrete-event simulation optimization using ranking, selection, and multiple comparison procedures: a survey." ACM Transactions on Modeling and Computer Simulation 13:134-154.

Teng, S., Lee, L. H., and Chew, E. P. 2010. "Integration of Indifference-zone with Multi-objective Computing Budget Allocation.” European Journal of Operational Research 203:419-429.

\section{AUTHOR BIOGRAPHIES}

NUGROHO A. PUJOWIDIANTO is an R\&D Writing System Engineer in the Business Printing Division, Printing and Personal Systems Group at Hewlett-Packard Singapore. He received his B.Eng. (Mechanical Engineering) degree from Nanyang Technological University in 2006 and his Ph.D. degree from the Department of Industrial and Systems Engineering, National University of Singapore in 2013. He is a member of IEEE and was previously a teacher at Pioneer Secondary School under the Ministry of Education, Singapore. His research interests include simulation optimization and its application in health care. His email address is nugroho@hp.com.

LOO HAY LEE is an Associate Professor and Deputy Head (Research) in the Department of Industrial and Systems Engineering, National University of Singapore. He received his B.S. (Electrical Engineering) degree from the National Taiwan University in 1992 and his Ph.D. degree in 1997 from Harvard University. He is currently a senior member of IEEE, a member of ORSS and INFORMS. He is the associate editor for IIE Transactions, Flexible Services and Manufacturing Journal, the Asia Pacific Journal of Operational Research, the co-editor for Journal of Simulation and is a member in the advisory board for OR Spectrum. His research interests include simulation-based optimization, and maritime logistics. His email address is iseleelh@nus.edu.sg.

CHUN-HUNG CHEN is a Professor in the Department of Systems Engineering and Operations Research at George Mason University. He received his Ph.D. from Harvard University in 1994. His research interests are mainly in development of very efficient methodology for simulation and optimization and its applications. Dr. Chen has served as Co-Editor of the Proceedings of the 2002 Winter Simulation Conference and Program Co-Chair for 2007 Informs Simulation Society Workshop. He is currently an associate editor of IEEE Transactions on Automatic Control, area editor of Journal of Simulation Modeling Practice and Theory, associate editor of International Journal of Simulation and Process Modeling, and simulation department editor for IIE Transactions. His email address is cchen9@gmu.edu and his web page is http://mason.gmu.edu/ cchen9. 